\title{
RADIAÇÃO ULTRAVIOLETA/ ÍNDICE ULTRAVIOLETA E CÂNCER DE PELE NO BRASIL: CONDIÇÕES AMBIENTAIS E VULNERABILIDADES SOCIAIS
}

\author{
OLIVEIRA, Marcia Maria Fernandes de - marfernandesoliveira@gmail.com \\ Doutora em Geografia pela Universidade Federal do Paraná.
}

\begin{abstract}
RESUMO: A Radiação Ultravioleta (R-UV) possibilita a síntese de vitamina D na pele humana; todavia, quando a exposição é elevada ela pode causar danos à saúde, como o câncer de pele. Para analisar a relação entre a dimensão espacial da R-UV (convertida em Índice Ultravioleta - IUV) e o câncer de pele no Brasil utilizou-se da concepção sistêmica, sendo que foram empregados dados da doença (INCA Instituto Nacional do Câncer) e dados de IUV (CEPETEC - INPE: Instituto Nacional de Pesquisas Espaciais). No estudo foram evidenciados os fatores que se associam na definição de um padrão de pessoas com maior risco de desenvolver câncer de pele (pele clara), tanto devido à condição ambiental (R-UV) e espacial (região Sul) quanto sócio-econômica (baixa renda) que, juntas, conduzem a maior vulnerabilidade de desenvolver a doença. Não foi realizada correlação entre IUV e dados de câncer de pele, devido ao fato dos efeitos dos raios solares serem cumulativos. O câncer de pele é um problema de saúde pública, sendo o conhecimento de suas variações espaciais um dado importante para sua prevenção e combate.

Palavras-chave: Radiação Ultravioleta - Índice Ultravioleta - Câncer de Pele - Brasil

ULTRAVIOLET RADIATION / UV INDEX AND SKIN CANCER IN BRAZIL: ENVIRONMENTAL AND SOCIAL VULNERABILITY

ABSTRACT: The Ultraviolet Radiation (UV-R) enables the synthesis of vitamin $D$ in human skin; however, when exposure is high it may cause damage to health, such as skin cancer. To analyze the relationship between the spatial dimension of the R-UV (Ultraviolet Index converted - UVI) and skin cancer in Brazil we used the systems analysis approach, with were used data of disease (INCA - National Cancer Institute) and data of UVI (CEPETEC - INPE: National Institute for Space Research). In the study the factors that are associated with the definition of a pattern of people at higher risk of developing skin cancer (fair skin), both due to (UV - R) and spatial environmental condition (Southern region) and socioeconomic were evidenced (low income), which together lead to greater vulnerability of developing the disease. No correlation between UVI and data of skin cancer, due to the fact the effects are cumulative from sunlight was performed. Skin cancer is a public health problem, with the knowledge of its spatial variation its an important data to prevent and combat it.
\end{abstract}

Key-words: Ultraviolet Radiation - Ultraviolet Index - Skin Cancer - Brazil.

\section{INTRODUÇÃO}

O Brasil é um país ensolarado praticamente durante todo o ano, uma característica marcante da sua tropicalidade; a população brasileira encontra-se, portanto e de maneira geral, exposta a médias e altas incidencias de radiação solar. Tomando se por base o senso comum, o efeito direto da ação da luz solar sobre a pele humana - o bronzeamento - significa aparência saudável. Todavia, do ponto de vista científico, a exposição humana aos raios solares é tema de consideráveis debates e preocupações.

Exposições cautelosas ao sol, no início da manhã e nas últimas horas da tarde, são benéficas, pois ativam a circulação sanguínea periférica e possibilita a síntese de vitamina $D$ na pele. Especialmente em crianças e jovens esse processo é de fundamental importância, pois a vitamina $D$ (antirraquítica) é indispensável para uma boa ossificação e, portanto, para um crescimento saudável. No entanto, devese considerar os riscos que a Radiação Ultravioleta (R-UV) em excesso pode causar à pele; esta, segundo Okuno e Vilela (2005), pode causar uma série de danos à saúde humana tais como o envelhecimento precoce, a depleção do sistema imunológico, a catarata e o câncer de pele, dentre outros.

Na década de 1990 Kirchhoff (1995) apontava os perigos da radiação danosa sobre a pele humana; segundo ele, evidências científicas mostravam que a radiação (particularmente a UVB) deveria aumentar sua intensidade com a destruição progressiva da camada de ozônio da estratosfera, e com isto elevar a incidência de câncer de pele na população. Após quase vinte anos, sua preocupação continua sendo coerente, pois percebe-se um aumento progressivo da incidência do câncer de pele na população, em especial na região Sul do Brasil (NASSER, 1986, 1993; 
AZEVEDO e MENDONÇA, 1992; CORRÊA, M. P.; DUBUISSON, P.; PLANAFATTORI, A. (2003).

Entre 2002 e 2005 o Ministério da Saúde (MS) realizou um inquérito domiciliar sobre o comportamento de risco do percentual de indivíduos (a partir de 20 anos) e pessoas expostos a R-UV por no mínimo 30 minutos (figura 01), concluindo que o percentual de pessoas no Sul do Brasil que se expõe a R-UV por mais de meia hora é muito elevado, acima de $75 \%$.

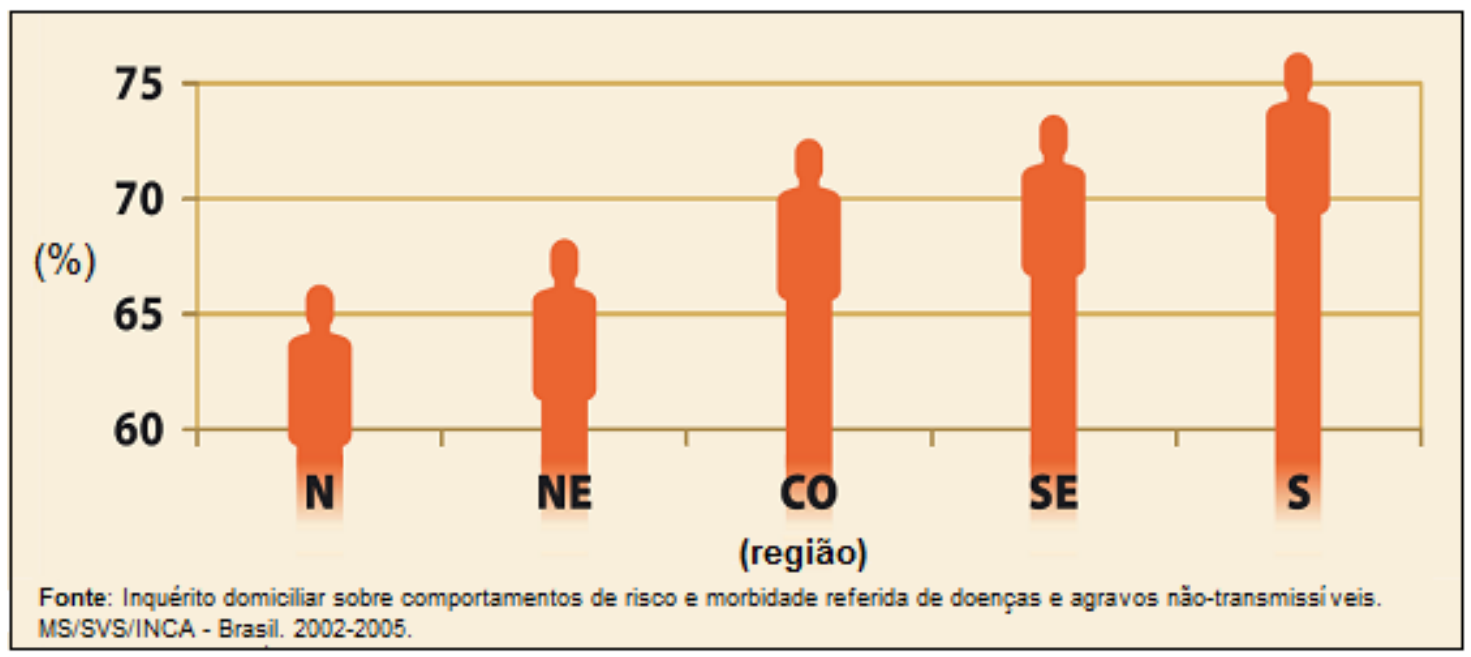

Figura 01. Percentual de indivíduos ( 20 anos ou mais) expostos à radiação solar por pelo menos 30 minutos por região do Brasil (2002-2005)

Segundo a Organização Mundial da Saúde - OMS (2006), "um em cada três casos de câncer diagnosticados no mundo corresponde ao câncer de pele", enquanto no Brasil, conforme o segundo o Instituto Nacional do Câncer - INCA, a estimativa é de 98.420 novos casos de câncer de pele não melanoma nos homens e 83.710 nas mulheres para 2014. Esses valores correspondem a um risco estimado de 100.75 casos novos a cada 100 mil homens e 82.24 a cada 100 mil mulheres. O câncer de pele não melanoma é o mais incidente em homens nas regiões Sul (159.51/100 mil), Sudeste (133. 48/100 mil) e Centro-Oeste $(110.94 / 100 \mathrm{mil})$; nas regiões Nordeste $(40.37 / 100 \mathrm{mil})$ e Norte $(28.34 / 100 \mathrm{mil})$, encontra-se na segunda posição. Nas mulheres é o mais frequente em todas as regiões, com um risco estimado de 112.28/100 mil no Sudeste, 99.31/100 mil Centro-Oeste, 86.03/100 mil no Sul, 46.68/100 mil no Nordeste e $24.73 / 100$ mil no Norte.

A relação entre a R-UV, aqui tratada sob a perspectiva do Índice Ultravioleta (IUV), e o câncer de pele no Brasil é o foco central deste texto. Para tanto, são colocados em evidencia importantes aportes teóricos ao tema e uma análise da variação espacial das duas variáveis, bem como da vulnerabilidade social ao câncer de pele no contexto brasileiro.

\section{MATERIAL E MÉTODO}

Para a elaboração deste estudo tomou-se por base uma estruturação na qual a metodologia de pesquisa tem como alicerce a concepção Sistêmica, pois esta tem como objetivo a formulação de princípios válidos para os "sistemas" em geral, qualquer que seja a natureza dos elementos que os compõem e as relações ou "forças" existentes entre eles. A Radiação Solar, o Índice Ultravioleta e a exposição humana a ela compreendem o input de nosso sistema, sendo que as condições sócio-etnicas e culturais da população os atributos, e o câncer de pele o output do mesmo. 
Este artigo foi desenvolvido em grande parte a partir de literatura específica ao tema e de pesquisas realizadas em instituições relacionadas à oncologia, com destaque ao Instituto Nacional do Câncer (INCA), principal fonte de dados de câncer de pele no Brasil. Os dados de R-UV convertidos em IUV foram obtidos via Centro de Previsão de Tempo e Estudos Climáticos e Instituto Nacional de Pesquisas Espaciais.

É fundamental ressaltar, segundo Fernandes de Oliveira (2010, pág. 16), entre IUV e câncer de pele não há possibilidade de correlação, pois os efeitos dos raios solares são cumulativos. Assim a análise entre os casos de câncer de pele e tais variáveis não busca relação direta de causa e efeito e sim do entendimento de como apresentam as tendências de cada um.

As análises foram realizadas por meio de relações estabelecidas entre o aporte da literatura específica, os dados de câncer de pele e os dados de IUV no Brasil; a abordagem tem como centro a dimensão espacial entre as diferentes variáveis envolvidas na pesquisa.

\section{RADIAÇÃO ULTRAVIOLETA}

A Radiação solar corresponde à emissão de energia sob a forma de ondas eletromagnéticas compreendidas entre $100 \mathrm{~nm}^{1}$ e acima de $800 \mathrm{~nm}$ que se propagam à velocidade da luz (KIRCHHOFF, 1995). Ela pode ser separada em três grupos, de acordo com o comprimento de onda. A parte mais diretamente relacionada ao aquecimento da atmosfera terrestre é aquela compreendida pelo Infravermelho, Visível e Ultravioleta. A faixa do ultravioleta é ainda subdividida em três (tabela 01): a UVA, entre 400 e 320 nm; a UVB, entre 320 e $280 \mathrm{~nm}$; e a UVC, entre 280 e $100 \mathrm{~nm}$.

Tabela 01. Faixa do Ultravioleta em UVC, UVB e UVA

\begin{tabular}{|l|c|l|}
\hline Nome & $\begin{array}{c}\text { Intervalo } \\
\text { espectral } \\
\text { (nm) }\end{array}$ & \multicolumn{1}{|c|}{ Características } \\
\hline UVC & $100-280$ & $\begin{array}{l}\text { Completamente absorvida pelo } \mathrm{O}_{2} \text { e } \mathrm{O}_{3} \text { estratosférico, } \\
\text { portanto, não atinge a superfície terrestre. É utilizada na } \\
\text { esterilização de água e materiais cirúrgicos. }\end{array}$ \\
\hline UVB & $280-320$ & $\begin{array}{l}\text { Fortemente absorvida pelo } \mathrm{O}_{3} \text { estratosférico. É prejudicial à } \\
\text { saúde humana, podendo causar queimaduras e, a longo } \\
\text { prazo, câncer de pele. }\end{array}$ \\
\hline UVA & $320-400$ & $\begin{array}{l}\text { Sofre pouca absorção pelo } \mathrm{O}_{3} \text { estratosférico. É importante } \\
\text { para sintetizar a vitamina D no organismo. Porém o excesso } \\
\text { de exposição pode causar queimaduras e, a longo prazo, } \\
\text { causa o envelhecimento precoce. }\end{array}$ \\
\hline
\end{tabular}

Fonte: http://satelite.cptec.inpe.br/uv/R-UV.html adaptado pela autora.

Kirchhoff (1995) afirma que a energia solar recebida pelo nosso planeta refere-se basicamente ao espectro visível do sol, cuja máxima intensidade esta perto de 500 $\mathrm{nm}$, isto é, na cor verde. A intensidade da radiação solar é várias ordens de grandeza menor, na região do UV, do que na faixa do visível. Mesmo assim, é nesta pequena faixa do espectro solar que se define a radiação que mais interfere com os sistemas biológicos. Na faixa de 280 para $320 \mathrm{~nm}$, a intensidade de radiação cresce rapidamente, ou seja, a intensidade é muito maior em 320 do que em $280 \mathrm{~nm}$; no entanto, a sensibilidade biológica se comporta ao contrário, isto é, ela é maior em $280 \mathrm{~nm}$, decrescendo rapidamente para o lado de $320 \mathrm{~nm}$. É esta

\footnotetext{
${ }^{1} \mathrm{~nm}$ é abreviação de nanômetro, que vale $10^{-9}$ metros
} 
variação da sensibilidade biológica que é chamada de espectro de ação, ou espectro de sensibilidade biológica.

Fatores temporais, geográficos e meteorológicos afetam a irradiância espectral da R-UV na superfície da Terra os quais, segundo Okuno e Vilela (2005) são:

a) Hora do dia: no verão, cerca de 20 a 30\% da irradiância total diária de R-UV atinge a Terra entre 11 e 13 horas, e entre 70 e $80 \%$ entre 9 e 15 horas.

b) Estação do Ano: a irradiância da R-UVB diária, próximo ao equador ( $20^{\circ}$ $\mathrm{N})$, apresenta variação sazonal de mais $25 \%$ no verão e menos $30 \%$ no inverno em relação à primavera/outono. Na zona temperada $\left(40^{\circ} \mathrm{N}\right)$, esses valores correspondem a mais $70 \%$ e menos $70 \%$ respectivamente.

c) Latitude geográfica: o fluxo da R-UV diminui com o aumento da distância ao Equador.

d) Altitude: em geral, a cada quilômetro de aumento na altitude, o fluxo de R-UV aumenta ao redor de $6 \%$.

e) Nuvem: a presença de nuvens no céu afeta muito a irradiância de radiação infravermelha, mas pouco a de R-UV. Se o sol estiver encoberto por nuvens, a quantidade de R-UVB ainda corresponderá a cerca de $50 \%$ daquela de um dia claro.

f) Reflexão na Superfície: a neve e a areia contribuem muito para a refletância - cerca de $30 \%$ e $25 \%$ da R-UV, enquanto as superfícies terrestre e marítima refletem menos de $7 \%$. Assim, se uma pessoa estiver sob um guarda-sol na praia, não recebe radiação solar direta, mas recebe a radiação refletida pela areia.

g) Ozônio: é o fator mais importante de absorção da R-UV, principalmente da R-UVB e R-UVC solar dirigida à superfície terrestre.

Há, todavia divergências no que concerne aos parâmetros acima, ou seja: a altitude parece realmente desempenhar um papel mais perceptível na radiação somente de 2 a $3 \mathrm{~km}$ onde a atmosfera é mais rarefeita; quanto às nuvens, parece ser mais correto considerar a nebulosidade, condição que encerra a maior parte da umidade atmosférica e que desempenha portanto maior efeito na radiação. Quanto à refletância da superfície os valores podem ser bem maiores, pois a neve fresca, por exemplo, apresenta albedo de até $80 \%$ (MENDONÇA e DANNI-OLIVEIRA, 2007).

\section{4. ÍNDICE ULTRAVIOLETA}

Tendo em vista a crescente incidência de câncer de pele na escala mundial, a Organização Mundial da Saúde (OMS), em colaboração com o Programa das Nações Unidas para o Meio Ambiente (UNEP), a Organização Meteorológica Internacional (OMM), a Comissão Internacional de Proteção às Radiações NãoIonizantes (IC-NIRP), decidiu desenvolver um projeto conjunto de proteção da população contra os efeitos danosos da R-UV. A proposta foi a de associar uma escala que chama-se Índice Ultravioleta (IUV) aos níveis de R-UV relevantes aos efeitos biológicos estabelecidos no ser humano, que pode ser usado e compreendido facilmente e, dessa forma, adotado e divulgado diariamente em boletins meteorológicos. Segundo Okuno e Vilela (2005) esta decisão baseou-se no fato de que $90 \%$ das ocorrências de câncer de pele do tipo não-melanoma se dão em peles tipos I e II, conforme listado na Tabela 03. Além disso, ao proteger esses grupos, os demais, com pele tipos III, IV, V e VI, estarão automaticamente protegidos. É importante, entretanto, lembrar que, se estes últimos indivíduos são menos suscetíveis a câncer de pele, o mesmo não se aplica quanto aos efeitos nos olhos e no sistema imune. 
Para este estudo a R-UV foi associada ao número IUV, pois segundo Corrêa (2003: 66):

A estimativa do período de tempo máximo de exposição ao sol envolve não só fatores geográficos e sazonais, mas principalmente uma série de fatores inerentes ao próprio ser humano, tais como a cor natural da pele, dos cabelos e dos olhos, o desenvolvimento dos processos de queimadura e bronzeamento, condições de saúde e alimentação, ingestão de medicamentos e, até mesmo, reações alérgicas. Considerando indivíduos saudáveis, cor natural da pele e a cor após exposição ao sol são fundamentais para se estabelecer um padrão médio da resposta biológica à radiação ultravioleta. Essa resposta biológica refere-se ao processo de formação de eritema (avermelhamento da pele) após exposição à certa dose de R-UV. Ou seja, o tempo máximo de exposição esta relacionado à dose mínima de radiação necessária para que ocorra avermelhamento da pele e, possivelmente lesões de natureza mais grave.

O IUV conforme CPTEC/INPE (2014) representa o valor de máxima intensidade diária da R-UV referente ao meio-dia solar. Como a cobertura de nuvens é algo muito dinâmico e variável, o IUV é sempre apresentado para uma condição de céu claro. Isto é, para ausência de nuvens que, na maioria dos casos, representa a máxima intensidade de radiação. É apresentado como um número inteiro e, de acordo com recomendações da OMS, esses valores são agrupados em categorias de intensidades (Tabela 02).

Tabela 02. Intensidade do Índice Ultravioleta

\begin{tabular}{|ll|}
\hline CATEGORIA & INDICE ULTRAVIOLETA \\
\hline BAIXO & $<2$ \\
\hline MODERADO & 3 a 5 \\
\hline ALTO & 6 a 7 \\
MUITO ALTO & 8 a 10 \\
EXTREMO & $>11$ \\
\hline Fonte: http://satelite.cptec.inpe.br/uv/O_que_e_IUV.html \\
\hline
\end{tabular}

O Centro de Previsão do Tempo e Estudos Climáticos (CPTEC) em conjunto com o Instituto Nacional de Pesquisas Espaciais (INPE) ${ }^{2}$ via site http://www.cptec.inpe.br/ disponibiliza diariamente o IUV para a América do Sul, Brasil e suas regiões; a figura 02 ilustra um exemplo destas informações.

\footnotetext{
${ }^{2}$ Além do CPTEC/INPE existem outros Centros de previsão do tempo que disponibilizam o IUV diário.
} 

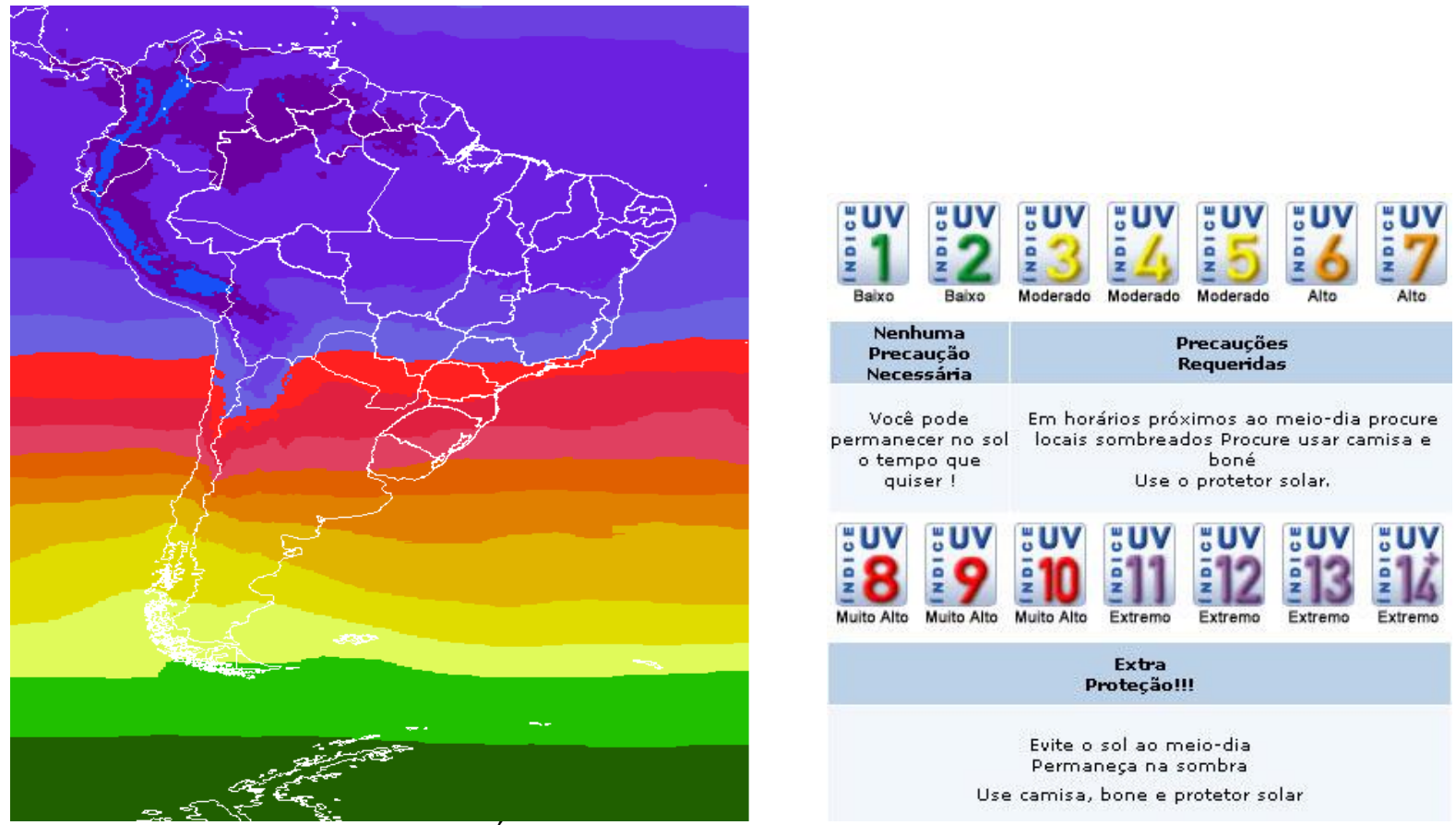

Figura 02.Máximo diário do Índice Ultravioleta para o Brasil e precauções da OMS.

Fonte: http://satelite.cptec.inpe.br/uv/ dia 30 de março de 2014.

A presença de nuvens e aerossóis (partículas em suspensão na atmosfera) atenua a quantidade de radiação UV em superfície, segundo o CPTEC - INPE (2014). Porém, parte dessa radiação não é absorvida ou refletida por esses elementos e atinge a superfície terrestre. Deste modo, dias nublados também podem oferecer perigo, principalmente para as pessoas de pele sensível.

A intensidade com que as radiações alcançam o solo é denominada de intensidade de insolação e está relacionada à altura solar de cada lugar, a estação do ano, a quantidade de tempo de brilho solar, a nebulosidade e, sobretudo a latitude são os principais responsáveis pela duração da insolação. Segundo Mendonça e DanniOliveira (2007: 38) "A intensidade de insolação apresenta seus maiores valores nas regiões tropicais, por volta dos $20^{\circ}$ de latitude em ambos os hemisférios". No entanto, além deste fator de posição latitudinal, o que influencia mais diretamente a alta insolação destas áreas $\left(20^{\circ}\right.$ a $40^{\circ}$ de latitude Norte e Sul) é a predominância do ar subsidente nestas localidades.

\section{CÂNCER DE PELE E RADIAÇÃO SOLAR}

Câncer, segundo o Instituto Nacional do Câncer (INCA, 2007) é o nome dado a um conjunto de mais de cem doenças que têm em comum o crescimento desordenado (maligno) de células que invadem os tecidos e órgãos, podendo espalhar-se para outras regiões do corpo. Os diferentes tipos de câncer correspondem aos vários tipos de células do corpo; por exemplo, existem diversos tipos de câncer de pele porque a pele é formada de mais de um tipo de célula. Se o câncer tem início em tecidos epiteliais, como pele ou mucosas, ele é denominado carcinoma; se começa em tecidos conjuntivos como osso, músculo ou cartilagem é chamado de sarcoma. Outras características que diferenciam os diversos tipos de câncer entre si são a velocidade de multiplicação das células e a capacidade de invadir tecidos e órgãos vizinhos ou distantes (metástases). 
As neoplasias cutâneas, segundo o INCA (2007) estão relacionadas a alguns fatores de risco, como o químico (arsênico), a radiação ionizante, processo irritativo crônico (úlcera de Marjolin ${ }^{3}$ ), genodermatoses (Xeroderma Pigmentosum ${ }^{4}$ etc) e principalmente à exposição às R-UV do sol.

Dois tipos de envelhecimento, segundo a Sociedade Brasileira de Dermatologia - SBD (2007), se processam na pele, o intrínseco e o extrínseco. O envelhecimento intrínseco ocorre com o passar dos anos, também chamado de envelhecimento cronológico; O extrínseco decorre da interação dos fatores ambientais com a pele, sendo que um dos fatores mais importantes no envelhecimento extrínseco é a exposição solar. Os raios ultravioletas são os maiores causadores de câncer de pele, envelhecimento precoce e aparecimento das queratoses solares. A exposição solar tem efeito cumulativo, podendo o câncer de pele surgir muitos anos mais tarde. Estudos recentes revelam que a proteção ao sol na infância e adolescência reduzem significativamente os riscos de câncer de pele, pois cerca de $80 \%$ de toda radiação solar que uma pessoa recebe durante toda a vida se concentra nos primeiros 18 anos de idade, exatamente a fase da vida onde a criança e o adolescente ficam grande parte ao ar livre.

Segundo o INCA (2009), como a pele é um órgão heterogêneo, esse tipo de câncer pode apresentar neoplasias de diferentes linhagens. Os mais frequentes são: carcinoma basocelular ( $\mathrm{CBC}$ ), responsável por $70 \%$ dos diagnósticos de câncer de pele, o carcinoma espinocelular (CEC) ou epidermóide com $25 \%$ dos casos e o melanoma, detectado em $4 \%$ dos pacientes. O carcinoma basocelular, o mais frequente, é também o menos agressivo; este, e o carcinoma espinocelular, são também chamados de câncer de pele não melanoma, enquanto o melanoma e outros tipos, com origem nos melanócitos, são denominados de câncer de pele melanoma (MM).

A proteção solar deve iniciar-se precocemente. A recomendação para uso de protetores solares se inicia aos 6 meses de idade, antes disso a criança não deve se expor diretamente ao sol sem a proteção de roupas e chapéu. Além disso, segundo o INCA (2009), os pacientes imunocomprometidos (como os transplantados renais) têm um maior risco para o desenvolvimento do câncer de pele não melanoma.

O CBC e o CEC, conforme a SBD (2007), são as neoplasias mais frequentes da pele e estão diretamente relacionados com exposições solares frequentes de pessoas de pele clara ao longo dos anos. As lesões ocorrem principalmente nas áreas mais fotoexpostas como face, pescoço, dorso, antebraços e mãos. Muitos deles poderiam ser evitados se medidas de prevenção fossem aplicadas a tempo, pois quando detectado, este tipo de câncer apresenta altos percentuais de cura.

Por outro lado, o câncer melanoma (MM) é um tipo especial de câncer de pele que vem apresentando um aumento importante em incidência e mortalidade no mundo inteiro, principalmente nos países nórdicos, Nova Zelândia e Israel, acometendo pessoas em idade mais jovem (15-34 anos), atingindo ambos os sexos. A

\footnotetext{
3 Segundo a Sociedade Brasileira de Dermatologia SBD (2009) http://www.anaisdedermatologia.org.br/public/artigo.aspx?id=713, pesquisa em 09/08/2009 às 17:49h) "Conceitua-se Úlcera de Marjolin como a neoplasia maligna que se origina de uma cicatriz, seja de queimadura ou seqüela de outras lesões tais como psoríase lúpus, sífilis, osteomielite, úlcera de estase, etc.

4 Conforme A Biblioteca Virtual em Saúde BVS (2009) http://bases.bireme.br/cgibin/wxislind.exe/iah/online/?IsisScript=iah/iah.xis\&src=google\&base =LILACS\&lang=p\&nextAction=Ink\&e xprSearch=335039\&indexSearch=ID, pesquisa em 09/08/2009 ás 17:57h O Xeroderma Pigmentosum é uma doença autossômica recessiva rara, caracterizada pelo desenvolvimento prematuro de neoplasias devido à extrema sensibilidade à radiação ultravioleta. Estas manifestações ocorrem por falha no mecanismo de excisão e reparo do DNA. Se comparados a indivíduos normais, estes pacientes apresentam risco 1000 vezes maior de desenvolver neoplasias em áreas expostas ao sol.
} 
exposição à luz solar tem sido relacionada como importante fator de risco. (HOSPITAL ERASTO GAERTNER - HEG, 2003). Configura-se como o mais agressivo e temido entre os tipos de câncer de pele, tendo sido também relacionado a exposições solares intensas, com queimaduras solares dolorosas e com bolhas, sobretudo durante a infância e adolescência. No entanto, o risco deste câncer não se restringe somente à exposição solar e pessoas de pele clara; apesar de raro ele pode acometer pessoas de pele morena e até negros. Pode surgir a partir da pele normal ou de uma lesão pigmentada.

\section{1. Efeitos da R-UV sobre a pele}

Quando a R-UV atinge a pele, parte é refletida de volta ao primeiro meio e a parte transmitida vai sendo absorvida pelas várias camadas até que a energia incidente seja totalmente dissipada (OKUNO e VILELA, 2005). Uma pequena fração da energia absorvida é reemitida como fluorescência. A absorção da R-UV varia muito com seu comprimento de onda. A radiação com comprimento de onda inferior a $315 \mathrm{~nm}$ (UVB + UVC) é em grande parte absorvida por proteínas e outros constituintes celulares epidérmicos, reduzindo muito a sua penetração na pele. $O$ remanescente é presumivelmente absorvido pelo DNA e outros componentes dérmicos: a elastina e o colágeno. A radiação com comprimento de onda superior a $315 \mathrm{~nm}$ (UVA) alcança a derme após absorção variável pela melanina epidérmica, a espessura da pele e seu teor de melanina interferem na absorção e difusão da radiação.

As reações fotobiológicas são mais intensas quanto mais precoce for o início da insolação e dependem do tempo e do número de exposições. Também influem a intensidade e o comprimento de onda da radiação que no caso, de R-UV solar, variam de acordo com a altitude, latitude, estação do ano, condições atmosféricas e hora do dia. Os efeitos biológicos consequentemente são agudos ou imediatos quando surgem após algumas horas ou alguns dias após a exposição, e crônicos ou tardios quando são consequência da somatória e exposições agudas repetitivas no decorrer da vida. A (figura 03) ilustra o processo da penetração das ondas de R-UV, A, B e C na pele humana.

A OMS (2007) esclarece como a R-UV, A e B atuam sobre a pele, respectivamente: Os raios UVA ativam a melanina e criam um bronzeamento que aparece rapidamente, mas que perde-se igualmente rápido. Além disso, os raios UVA penetram nas camadas profundas da pele e afetam o tecido conjuntivo e os vasos sanguíneos, sendo que a pele começa a enrugar-se. Os raios UVB estimulam a produção de nova melanina, o que conduz a um aumento maciço das quantidades de pigmento escuro em alguns dias; este bronzeamento pode durar relativamente muito tempo. Os raios UVB estimulam igualmente as células de modo que produzam uma epiderme mais espessa. Assim, os raios UVB são responsáveis pelo enfraquecimento e espessamento das camadas superficiais da pele - reações que constituem o mecanismo de defesa do organismo contra os raios ultravioletas. 


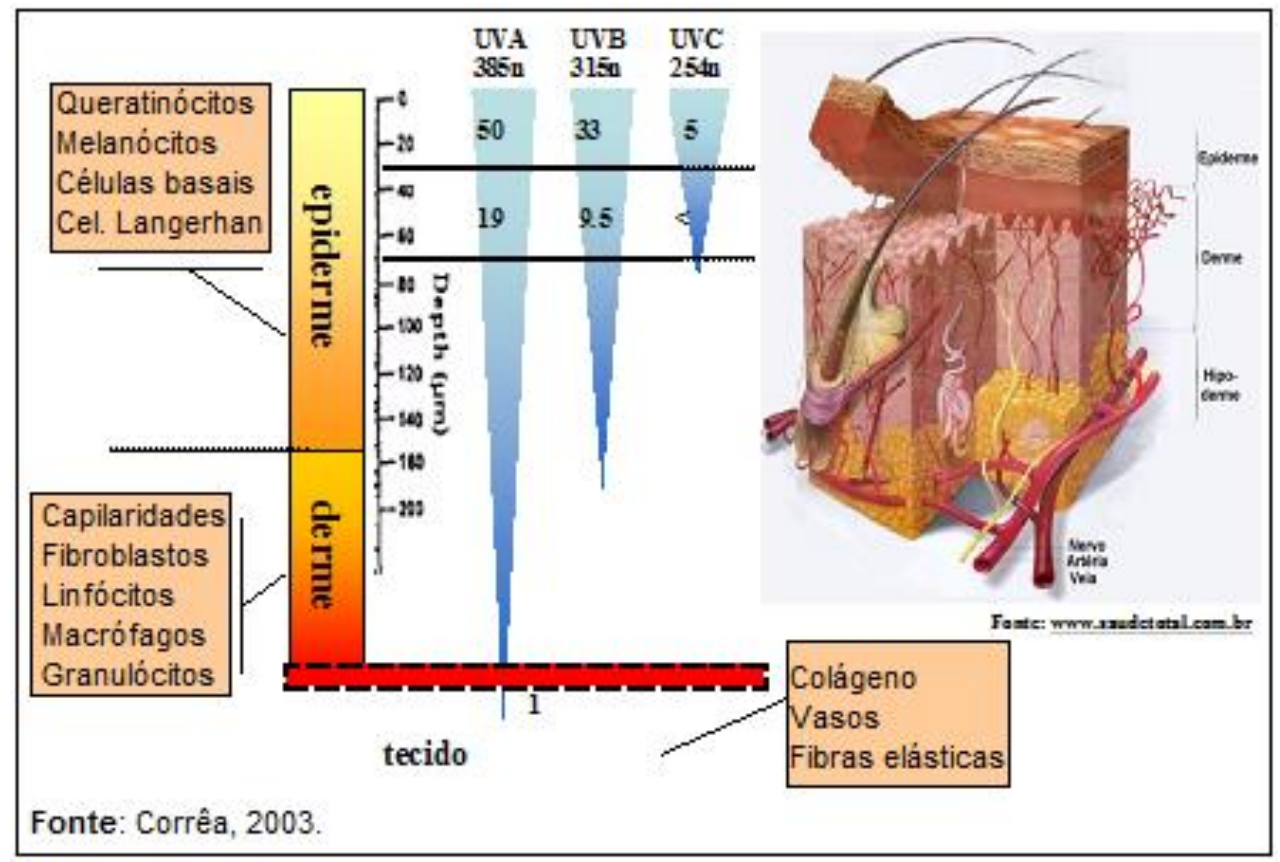

Figura 03. Radiação Ultravioleta na pele Humana Fonte: Corrêa, 2003.

\section{2. 1. Radiação Ultravioleta e Câncer de Pele}

Desde a década de 1930, segundo Corrêa (2003), diversos estudos foram realizados no intuito de estabelecer uma relação entre o tipo de pele e a dose mínima de radiação - ou Dose Eritematosa Mínima - DEM (Ürbach, 1969). A classificação de Fitzpatrick (1988) apud. Corrêa (2003), apresentada na tabela 3 e utilizada como referência por grande parte dos dermatologistas, é resultado de experimentos realizados com centenas de indivíduos de diferentes tipos de pele submetidos à exposição a quantidades controladas de R-UV. Nessa tabela, são apresentados valores da DEM (em $\mathrm{mJ} \mathrm{cm}^{-2}$ ) de acordo com cada tipo de pele e com reação à exposição; vale ressaltar que essa caracterização da reação da pele tende a ser subjetiva, uma vez que, sob condições reais, a vulnerabilidade da pele de um indivíduo particular depende de seus hábitos de exposição (manhã, tarde, verão, inverno, etc.), do tempo de exposição acumulado ao longo dos anos, de sua idade, de suas condições de saúde e de sua alimentação. Como principal evidência podese observar que indivíduos com pele mais clara necessitam de uma dose de radiação menor para desencadear um processo eritêmico, em relação àquelas observadas por indivíduos de pele mais escura. Por esta razão, a incidência de doenças relacionadas à exposição à R-UV é muito maior em indivíduos de pele branca, cabelos e olhos claros do que em mulatos e negros (Diffey apud Corrêa, 2003).

Tabela 03. Tipos de Pele e Reação a Exposição à Radiação UVB

\begin{tabular}{|c|c|c|c|c|}
\hline $\begin{array}{c}\text { Cor da pele } \\
\text { sem } \\
\text { exposição }\end{array}$ & $\begin{array}{c}\text { Tipos de } \\
\text { pele }\end{array}$ & Queimadura & Bronzeamento & $\begin{array}{c}\text { DEM* } \\
\mathbf{m J} / \mathbf{c m}^{2}\end{array}$ \\
\hline \multirow[t]{4}{*}{ Branca } & $\mathrm{I}$ & Sim & Não & $20-30$ \\
\hline & II & Sim & Mínimo & $25-35$ \\
\hline & III & Sim & Sim & $30-50$ \\
\hline & IV & Não & Sim & $45-60$ \\
\hline Mulata & $\mathrm{V}$ & Não & Sim & $60-100$ \\
\hline Negra & VI & Não & Sim & $100-200$ \\
\hline
\end{tabular}


Fonte: (Adaptada de Fitzpatrick, apud Corrêa, 2003).

\section{O CÂNCER DE PELE NO BRASil: VARIAÇÃo ESPACIAL E VULNERABILIDADE SOCIAL}

Dos tumores existentes, o câncer de pele é o mais frequente; para todos os tipos de câncer de pele, os fatores de risco incluem: histórico de câncer de pele na família, sensibilidade da pele ao sol, história de exposição solar excessiva, doenças imunossupressoras e exposição ocupacional. INISTITUTO NACIONAL DO CÂNCER INCA (2010) e SOCIEDADE BRASILEIRA DE DERMATOLOGIA - SBD (2009).

O INCA (2009) aponta o câncer de pele como o mais frequente no país (figura 04), correspondendo a aproximadamente $25 \%$ de todos os tumores diagnosticados em todas as regiões do Brasil, mas com maior incidência na região Sul, onde existe uma maior concentração de pessoas de pele clara, mais vulneráveis a incidência de câncer de pele. Os mesmos dados revelam que o câncer que mais acomete os brasileiros é o de pele não melanoma (CPNM) os basocelulares (CBC) e os espinocelulares (CEC) ou células escamosas; em segundo aparecem os cânceres de mama e o de próstata, respectivamente relacionados com o gênero feminino e masculino.

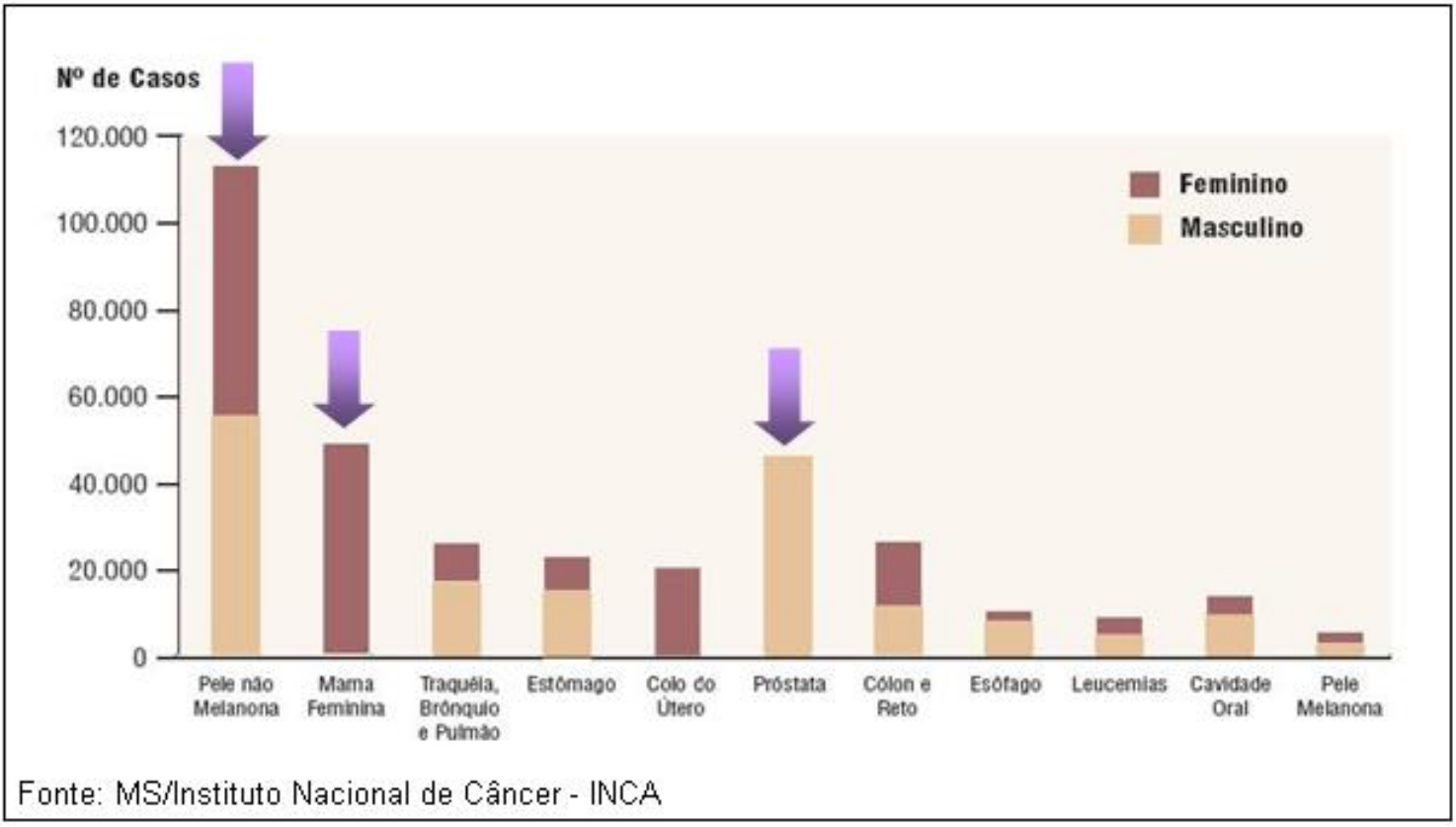

Figura 04. Câncer no Brasil

Ainda conforme o INCA (2010), no Brasil, o câncer de pele continua sendo o tipo mais incidente para ambos os sexos. Sua letalidade é considerada baixa, porém em alguns casos onde há demora no diagnóstico esse câncer pode levar a ulcerações e deformidades físicas graves. É quase certo que exista um considerável sub-registro devido ao subdiagnóstico e também por ser uma neoplasia de excelente prognóstico, com taxas altas de cura completa, se tratada de forma adequada e oportuna.

O Melanoma (MM) é menos frequente do que os CPNM, porém sua letalidade é mais elevada. Tem-se observado um expressivo crescimento, segundo o INCA (2009) na incidência deste tumor em populações de cor de pele branca. O prognóstico do MM pode ser considerado bom, se detectado nos estágios iniciais; nos últimos anos 
houve uma grande melhora na sobrevida dos pacientes com este tipo de câncer, principalmente devido à deteç̧ão precoce do mesmo. Nos países desenvolvidos a sobrevida média estimada em cinco anos é de $73 \%$, enquanto que, para os países em desenvolvimento a sobrevida média é de $56 \%$. A média mundial estimada é de $69 \%$. INCA (2010)

Segundo a OMS (2007) estes dados são agravantes quando a escala global é colocada em pauta, pois:

A cada ano, no mundo, ocorre entre 2 e 3 milhões de cânceres de pele não melanoma e mais de 130.000 melanomas malignos. Mudanças no comportamento e a exposição ao sol são em grande parte responsáveis pelo aumento dos cânceres cutaneos.

Segundo o Instituto Brasileiro de Controle do Câncer - IBCC (2008), a Austrália é o local que registra a maior incidência de CPNM. Enquanto no Brasil são mais de um milhão de novos casos de cânceres variados por ano, sendo que inúmeros nem sequer são registrados devido à subnotificação, ou seja, não há registro por acometerem pessoas residentes em remotos lugarejos, que falecem, vitimas de neoplasia maligna, sem que este fato conste de seus atestados de óbitos (BARBOSA, 2003).

Ao se referir à espacialização geográfica dos casos de câncer de pele, Corrêa et. al. (2003) apontam para o fato de que parece estar associada à distribuição da população, com maior incidência da doença no sul e sudeste do país, onde concentra a maior parte da população branca, que coincide com o pensamento de Silva Mendonça (1992), pois, a evidência de que pessoas de pele clara têm maior predisposição para o desenvolvimento deste tipo de câncer é fortalecida ao se verificar que os coeficientes de ocorrência, em populações de pele negra e amarela, são inferiores às de pele branca e que, há no país populações de maior risco para o câncer de pele, em geral, representadas por descendentes de europeus. Nas regiões sudeste e sul, onde foi mais intensa a concentração de imigrantes da Europa Central, existem comunidades que, por razões geográficas, sociais e culturais sofreram pouca ou quase nenhuma miscigenação racial. Consequentemente, estas comunidades expressam risco importante, por suas características genéticas, para o desenvolvimento de câncer de pele.

Câncer de pele é mais comum, segundo o INCA (2010) em indivíduos com mais de 40 anos sendo relativamente raro em crianças e negros, com exceção daqueles que apresentam doenças cutâneas prévias. Indivíduos de pele clara, sensível à ação dos raios solares, ou com doenças cutâneas prévias são as principais vitimas do câncer de pele. Os negros normalmente têm câncer de pele nas regiões palmares e plantares.

Conforme o Hospital Erasto Gaertner - HEG (2006) referencia de tratamento oncológico no sul do Brasil, o CBC e o CEC são os tumores mais comuns no Brasil como em vários outros países, com 55.480 novos casos de câncer de pele não melanoma em homens e de 61.160 em mulheres em 2006. O risco foi, portanto de 61 novos casos a cada 100 mil homens e 65 para cada 100 mil mulheres. A incidência de CBC é maior em relação ao CEC na razão aproximada de 4:1 e corresponde a aproximadamente $75 \%$ dos casos de câncer da pele.

Cabe mencionar a estimativa do INCA para o ano de 2014, onde: Esperam-se 98.420 casos novos de câncer de pele não melanoma nos homens e 83.710 nas mulheres no Brasil. Esses valores correspondem a um risco estimado de 100,75 casos novos a cada 100 mil homens e 82,24 a cada 100 mil mulheres. 
Observa se a partir dos dados do HEG (2006) e Estimativa do INCA (2014) um considerável aumento no risco de se contrair a doença tanto em decorrência do aumento populacional quanto pela maior vulnerabilidade da população frente às situações ambientais e socioeconômicas.

Ainda segundo a Estimativa do INCA (2014), o câncer de pele não melanoma é o mais incidente em homens nas regiões Sul $(159,51 / 100$ mil), Sudeste $(133,48 /$ $100 \mathrm{mil})$ e Centro-Oeste $(110,94 / 100 \mathrm{mil})$. Nas regiões Nordeste (40,37/ $100 \mathrm{mil})$ e Norte (28,34/ 100 mil), encontra-se na segunda posição. Nas mulheres, é o mais frequente em todas as regiões, com um risco estimado de 112,28/ 100 mil no Sudeste, 99,31/ 100 mil no Centro-Oeste, 86,03/ 100 mil no Sul, 46,68/ 100 mil no Nordeste e 24,73/ 100 mil no Norte.

Quanto ao melanoma, sua letalidade é elevada, porém sua incidência é baixa (2.960 casos novos em homens e 2.930 em mulheres). As maiores taxas estimadas em homens e mulheres encontram-se na região Sul.

\section{CONSIDERAÇÕES SINTETIZADORAS}

Os malefícios que a R-UV pode exercer nos seres humanos, podem ser provindos, inclusive das situações socioeconômicas. Para tanto os estudos que envolvem desse tipo de radiação configuram-se de extrema importância, pois, além do conhecimento de fenômenos climáticos e meteorológicos, também visa compreender se há uma incidência maior deste câncer em pessoas com condições financeiras desfavorecidas. Pearce (1997: 121) afirma que "Na maioria dos países industrializados, os estudos tem encontrado reiteradamente forte associação entre classe social e câncer, com risco relativo quase duas vezes maior quando se compara o grupo menos favorecido com o mais favorecido".

A abordagem sistêmica em muito favorece a compreensão das relações existentes entre IUV e câncer de pele. Neste estudo, o risco está relacionado à questão temporo- espacial, particularmente à exposição a R-UV e a vulnerabilidade das questões sociais em que os pacientes acometidos pelo câncer estão inseridos. A vulnerabilidade social tem sido utilizada para caracterizar grupos socialmente vulneráveis, ou seja, indivíduos que, por determinadas características ou contingências, são menos propensos a uma resposta positiva mediante algum evento adverso. Confalonieiri (2008) afirma que a aplicação do conceito de vulnerabilidade é fundamental para o mapeamento das populações sob maior risco de serem atingidas e, consequentemente, a tomada de decisão acerca de medidas de adaptação ou proteção da população contra os efeitos deletérios do clima na saúde.

O INCA (2009) chama atenção para o câncer de pele como o mais frequente na população Brasileira e destaca o Sul do Brasil como região de maior incidência, o que pode estar associado ao fato desta região apresentar uma maior concentração de pessoas de pele clara. Segundo Silva Mendonça, (1992), não há duvida de que os indivíduos de origem européia que vivem em clima tropical ou temperado apresentam um risco elevado de desenvolver câncer cutâneo. Grande parte dos imigrantes europeus que se estabeleceram no Brasil e seus descendentes se dedicaram ao trabalho rural. É possível encontrar-se nas regiões sudeste e sul comunidades agrícolas com grande concentração de pessoas de pele clara; esses indivíduos expõem se ao sol continuadamente, muitas vezes desde a primeira infância. É fundamental também destacar que, além das condições socioeconômicas e culturais, a desinformação da população quanto aos cuidados básicos e aos métodos de prevenção respondem pelas altas taxas do câncer de pele no Brasil. 
A prevenção do câncer de pele inclui a prevenção primária de proteção à exposição solar nociva desde a infância, educação e treinamento dos profissionais da saúde para o diagnóstico e muitas campanhas de prevenção.

Como o melanoma é um tipo de câncer pouco frequente se comparado a outros tipos como o de pulmão ou de mama, as medidas preventivas terão mais êxito se forem dirigidas diretamente aos grupos de maior risco. Torna-se, assim, necessário a condução de estudos no Brasil que possam identificar estes grupos de alto risco e a partir de então estabelecer-se política de controle que contemple medidas adequadas e específicas.

Diante deste estudo acredita-se que deve haver uma maior sensibilização das pessoas frente à seriedade inerente ao câncer de pele. Sendo assim, sugere-se que haja maior atenção junto à vigilância e as práticas educativas e preventivas do câncer de pele na atenção básica no Sistema Único de Saúde (SUS); maior propaganda e esclarecimentos sobre filtro de proteção solar e a necessidade de sua utilização; bem como redução dos seus valores para que grande, senão toda parcela da população tenha acesso; incentivo e promoção de estudos e pesquisas, inclusive entre instituições de outros países, que visem maior conhecimento da incidência do câncer de pele no Brasil; e que as campanhas contra o câncer de pele sejam mais agressivas e mais fortemente divulgadas; disponibilização mais rápida de exames de pele, sobretudo para a parcela mais carente da população; incentivo a projetos e divulgação nas redes de ensino, sobretudo, desde as séries inicias. 0 câncer de pele é um problema de saúde publica e é urgente a necessidade de adoção de medidas preventivas mais eficazes ao seu combate.

\section{REFERENCIAS BIBLIOGRÁFICAS}

AZEVEDO, G. e SILVA MENDONÇA. Risco crescente de melanoma de pele no Brasil. Revista de Saúde Pública (Scielo), vol. 26, n. 04. São Paulo, 1992.

BARBOSA, A. Câncer - Direito e Cidadania, $10^{\circ}$ ed. Ed. Arx. São Paulo, 2003.

CPTEC - Centro de Previsão de Tempo e Estudos Climáticos/ INPE - Instituto Nacional de Pesquisas Espaciais. http://www.cptec.inpe.br/ 2014.

CONFALONIEIRI, U. Mudança climática global e saúde humana no Brasil. In Parcerias Estratégicas - Mudança do clima no Brasil: vulnerabilidade, impactos e adaptação. Centro de Gestão e Estudos Estratégicos (CGEE) n. 27, Brasília/DF, 2008.

CORRÊA, M. de P. Índice Ultravioleta: Avaliações e Aplicações. Tese de Doutorado. Universidade de São Paulo, São Paulo, 2003.

CORRÊA, M. P.; DUBUISSON, P.; PLANAFATTORI, A. An overview of the ultraviolet index and the skin cancer cases in Brazil. Photochemistry and Photobiology, v. 78, n. 1, p. 4954, 2003.

FERNANDES DE OLIVEIRA, M. M. Índice Ultravioleta e Câncer de Pele no Estado do Paraná. Tese de Doutorado. Universidade Federal do Paraná, Curitiba/PR, 2010. HOSPITAL ERASTO GAERTNER - 10 anos de Registro Hospitalar de Câncer do Hospital Erasto Gaertner (1990 - 1999), Curitiba/PR, 2003. HOSPITAL ERASTO GAERTNER, Curitiba/PR, 2006.

IBCC-Instituto Brasileiro de Controle do Câncer. Em http://www.ibcc.org.br/indexSite.htm, pesquisado em 07/02/2008, 11:50h.

INCA, Instituto Nacional de Câncer - Ministério da Saúde, São Paulo/SP, 2007.

INCA, Instituto Nacional de Câncer - Ministério da Saúde, São Paulo/SP, 2009.

INCA, Instituto Nacional de Câncer - Ministério da Saúde, São Paulo/SP, 2010.

INCA, Instituto Nacional de Câncer - Ministério da Saúde, São Paulo/SP, 2014.

INMET, Instituto Nacional de Meteorologia. Brasília/DF, 2007.

KIRCHHOFF, H. J. W.V. Ozônio e Radiação UV-B. Transitec Editorial, São José dos Campos/SP. 1995.

MENDONÇA, F. e DANNI-OLIVEIRA. Climatologia - noções básicas e climas do Brasil. Ed. Oficina de textos. São Paulo, 2007.

NASSER, N. Incidência de câncer de pele na região Sul do Brasil. Biblioteca Virtual em Saúde 61 (2): 69-72, mar.- abr. 1986: http://bases.bireme.br/cgi- 
in/wxislind.exe/iah/online/?IsisScript $=i a h / i a h . x i s \& s r c=$ google\&base $=$ LILACS\&lang $=p$ \&nextAction $=$ Ink\&exprSearch $=34110$ \&indexSearch $=$ ID

OKUNO, E. e VILELA, C. A. P. Radiação Ultravioleta: Características e Efeitos. Ed. Livraria da Fisica. São Paulo, 2005.

Organização Mundial da Saúde, 2006.

Organisation Mondiale de la Santé - OMS. Les effets connus de UV sur la santé, em http: www.who.int/uv/faq/uvhealtfac/fr/print.html, 2007.

PEARCE, N. Classe Social e Câncer. In Equidade e Saúde- Contribuições da Epidemiologia. Ed. Fiocruz. Rio de Janeiro/RJ, 1997.

SBD - Sociedade Brasileira de Dermatologia - São Paulo/SP 2007.

SBD - Sociedade Brasileira de Dermatologia - São Paulo/SP 2009.

SILVA MENDONÇA, A. G. Risco crescente de melanoma de pele no Brasil. Revista de Saúde Publica (Scielo), vol. 26, n. 04. São Paulo, 1992.

http://www2.inca.gov.br/wps/wcm/connect/tiposdecancer/site/home/pele_nao_melanoma.

http://www.inca.gov.br/estimativa/2014/estimativa-24012014.pdf 